\title{
LA PESTE PORCINE AFRICAINE, L'AUTRE CRISE SANITAIRE
}

\author{
AFRICAN SWINE FEVER, THE OTHER SANITARY CRISIS
}

Michel RIEU ${ }^{1}$ et André JESTIN ${ }^{2}$

(Communication présentée le 4 février 2021)

\section{RÉSUMÉ}

La séance bi-académique entre I'Académie d'Agriculture de France (AAF) et l'Académie Vétérinaire de France (AVF) du 3 février 2021 était dédiée à la Peste Porcine Africaine (PPA). Les auteurs présentent le contexte de cette initiative, la problématique abordée puis introduisent les conférenciers biologistes, experts de la maladie, et économistes, experts des marchés en Europe et en Chine, pour terminer par une synthèse des conclusions de la séance.

Mots-clés : Porc, Sanglier, Phacochère, Peste Porcine Africaine, PPA, Asfarvirus, ornithodoros

\begin{abstract}
-ABSTRACT
The bi-academic thematic session organised by the French Academy of Agriculture (AAF) and the French Veterinary Academy (AVF), the 3rd of February 2021, was dedicated to African Swine Fever (ASF). The organisers introduced the global context of the threat, the objectives of the conference and presented the speakers; biologists, experts of the disease, economists, experts in trade and markets in Europe and China, and a synthesis of the session's conclusions.

Key words: Pig, Wild boar, Warthog, African Swine Fever, ASF, Asfarvirus, Ornithodoros
\end{abstract}

\section{LE CONTEXTE, LA PROBLEMATIQUE, LES CONFERENCIERS}

Les deux académies, qui ont déjà organisé une séance bi-académique en avril 2013 sur l'élevage de montagne, ont souhaité organiser une nouvelle séance sur une épizootie qui préoccupe les autorités de par son impact sanitaire et économique. Jacques Brulhet et Jean-Pierre Jégou, Présidents respectifs de l'AAF et de l'AVF, ont exprimé tout l'intérêt qu'ils portaient à l'initiative bi-académique favorisant les échanges et les débats.

La PPA, qui n'est pas zoonotique, est une maladie mortelle pour les porcs. D'origine africaine, elle se retrouve maintenant dans l'est de l'Europe et en Asie et tout particulièrement en Chine. De nombreux facteurs tels son épidémiologie complexe avec les réservoirs (sangliers), son potentiel endémique par l'existence d'arthropodes vecteurs (tiques molles) (Bernard et al. 2016) et la très grande résistance du virus dans l'environnement représentent de véritables défis pour le contrôle de la maladie. Aucun vaccin efficace n'est disponible pour l'instant et ceci malgré de nombreuses études fondamentales et appliquées orientées vers la mise au point d'outils d'induction d'une immunité protectrice (Lacasta et al. 2014 ; VACDIVA, 2019). Cet échec trouve son origine dans la méconnaissance de la structure et de la biologie du virus (Karger et al. 2019 ; Blue print, 2017) et du type d'immunité induite. Les travaux de recherche fondamentale dans l'objectif de définir le rationnel de la production de vaccins PPA protecteurs et d'élaboration des programmes de vaccination efficaces restent une priorité. La PPA s'est installée dans l'est de l'Europe jusqu'en Allemagne et une extension vers l'ouest est prévisible, alors que des cas ont été observés dans l'est de la Belgique, la France ayant été jusque-là épargnée. Il est donc très légitime pour les deux académies de programmer une séance thématique sur une émergence territoriale potentielle d'une maladie virale porcine à impact élevé sur le plan sanitaire. En ce qui concerne l'évolution 
en Chine, la situation est alarmante. La Covid a occulté cette autre crise sanitaire causée par la PPA. Il est légitime pour l'Académie vétérinaire de présenter la singularité de la situation épidémiologique en Chine. Cette analyse est à mener en cherchant à établir des similitudes entre les conditions de la gestion de la crise Covid et celle de la PPA.

L'objectif de cette séance thématique était de faire le point sur la situation en Europe et en Chine, sur l'état des connaissances sur le virus, son épidémiologie, mais aussi sur l'impact de cette maladie animale non zoonotique sur l'économie mondiale. Pour cela quatre conférenciers ont accepté de venir nous présenter leurs points de vue pour éclairer le débat. La première conférencière, Marie-Frédérique Le Potier, a évoqué la PPA en Europe, le virus, les vecteurs, l'épidémiologie de la maladie et les méthodes de lutte. Marie-Frédérique Le Potier est Chef d'Unité Virologie Immunologie Porcine, Responsable du laboratoire national de référence PPA à l'Anses Ploufragan. Elle a été présidente de GARA, l'Alliance Internationale de Recherche sur la PPA et a organisé le congrès international en France en 2016. Elle est experte à l'OIE pour les viroses porcines dont la PPA, et a travaillé avec son équipe à la mise au point de vaccins candidats contre la PPA en collaboration avec le centre de référence de Pirbright (Surrey, Angleterre) et avec les équipes européennes.

Le second conférencier, Jean-Christophe Audonnet (Boehringer-Ingelheim), a présenté la PPA en Chine, l'autre crise sanitaire, les méthodes de lutte et le contexte politique. Il a travaillé à Virogenetics aux USA et a réalisé les premiers travaux sur les vaccins à base d'un vecteur viral, le Canarypox (La technologie ALVAC). Il a été le directeur du laboratoire de recherche de Rhône-Mérieux, puis Mérial. En 2013, il pilote la stratégie de la recherche Europe et Asie de Mérial, est nommé responsable du programme Merial-Chine et est responsable du site de Singapour. Il est membre du comité scientifique de Bioaster, de Lyon Biopole.

Jean-Marc Chaumet est le troisième conférencier, il nous a parlé de la PPA en Chine, ses effets sur la production porcine et les flux d'importations, dans le contexte institutionnel et économique. Jean-Marc Chaumet est chef de projet au département Économie d'Idele (Institut de l'Élevage), spécialiste de la Chine. Il est en charge de la conjoncture laitière et mène des études sur les marchés et les politiques des produits bovins (lait et viande). Il est également rédacteur en chef de la lettre de veille sur les productions animales en Chine (Chine_Abcis) et poursuit des recherches sur la politique agricole chinoise. Il a co-écrit le livre " La Chine au risque de la dépendance alimentaire (PUF 2017)", avec Thierry Pouch, membre de l'Académie d'Agriculture.

Le quatrième conférencier, Boris Duflot, nous a parlé des effets induits, constatés ou projetés, sur les filières porcines dans le monde et sur les autres filières animales. Boris Duflot est directeur du Pôle Économie de l'Ifip-Institut du Porc, spécialiste de l'élevage et des filières porcines, il conduit avec ses collègues de l'IFIP des travaux de veille et des études sur la performance économique et les évolutions des filières en France et à l'international. Il est aussi animateur du RMT Filarmoni, qui traite de l'économie de toutes les filières alimentaires et délégué général
d'Abcis, un bureau d'étude constitué par les instituts techniques de productions animales.

\section{L'ENSEIGNEMENT DES BIOLOGISTES ET DES ECONOMISTES}

Inéluctables épizooties?

L'avancée de la Peste Porcine Africaine dans toutes les régions d'Europe est-elle « inéluctable », comme l'a évoqué Boris Duflot? C'est une perspective inquiétante dans un contexte où des scénarios mettent en avant les risques de multiplication des zoonoses et des pandémies. Ainsi, la Plateforme intergouvernementale scientifique et politique sur la biodiversité et les services écosystémiques fait le diagnostic, dans un rapport récent (IPBES, 2020), d'un lien indissociable entre la multiplication des zoonoses (maladies animales qui se transmettent à l'homme) et la dégradation de la nature sous l'action de l'Homme. S'il ne nous appartient pas de juger ici de la crédibilité de ces différents scénarios ou de leur degré de probabilité, on ne peut les ignorer complètement et on doit imaginer des moyens de prévention et de lutte.

Les moyens de contrer la progression

Si la Peste Porcine Africaine n'est pas une zoonose, elle a déjà impacté la vie des humains. Elle a provoqué d'énormes dégâts économiques et a profondément perturbé l'élevage et son commerce dans la vague épidémique qui touche l'Europe et l'Asie depuis 2007. Marie-Frédérique Le Potier nous a rappelé le long épisode ibérique auquel il n'a pu être mis fin que grâce à une mobilisation importante de moyens par la CEE après l'adhésion de l'Espagne et du Portugal dans les années 80 (Bech-Nielsen et al. 1995). Et à cette période encore, la Peste Porcine Africaine compte parmi les innombrables fléaux qui ont frappé Haïti, entrainant l'abattage de tout le troupeau paysan, qui était vital pour les populations rurales (Ebert, 1985). Pour combattre la maladie et prévenir ses effets, la mobilisation des moyens importants, publics et privés, reste donc nécessaire. Les tentatives de mise au point de vaccins efficaces ont échoué jusque-là, malgré de nombreux efforts de recherche. Sans faire de parallèle injustifié, on a vu à quelle vitesse la recherche vaccinale contre le SARS-CoV-2 s'est déployée, explorant de nouvelles pistes technologiques. L'action des autorités sanitaires publiques dépend des moyens dont disposent les Etats. Dans la période récente, on a vu la rapidité de réaction et l'efficacité de la République tchèque. Par contre, en Ukraine, en proie à des difficultés politiques, économiques ou «diplomatiques ", l'Etat est largement dépourvu de capacités d'intervention et la situation reste hors de contrôle. A l'échelle des unités d'élevage, ce sont les principes et les règles de la biosécurité qu'il faut mettre en œuvre. Il s'agit d'investissements dans des installations et de protocoles pour interdire certains accès et limiter les échanges avec l'extérieur au strict nécessaire.

\section{La mobilité comme un facteur de risques}

Les questions sanitaires nous confrontent à la question des mobilités, celle des produits, des véhicules, des animaux, des êtres humains et ce, à de multiples échelles, du local au global. 
Si on parle beaucoup, ces temps-ci, de gestes barrières et de confinement, les règles de biosécurité conduisent aussi à construire des barrières et à contrôler strictement l'accès aux élevages. Mais même si les élevages sont le plus possible fermés, les mouvements de camions restent fréquents (apport d'aliments, enlèvements d'animaux...). Et des personnes entrent et sortent des élevages, non sans contraintes quand les précautions sont prises, avec douche à l'entrée et à la sortie pour le personnel, et lavage des camions dans des espaces appropriés. Dans certains grands élevages et dans certains pays, comme on le voit en Chine, le personnel doit rester dans l'enceinte de l'élevage plusieurs semaines, pour limiter les contacts entre l'intérieur, réputé propre, et l'extérieur, vu comme contaminé, contaminant, ou à risque. Les collaborateurs sont assignés à résidence, en quelque sorte, ce qui n'est pas particulièrement un facteur d'attractivité du métier.

\section{Les objectifs des politiques publiques}

Comme l'a montré Jean-Marc Chaumet, en Chine, l'épidémie de Peste Porcine Africaine a mis en balance, voire en contradiction, plusieurs objectifs et préoccupations de politique publique. La souveraineté alimentaire nationale est un souci historique et pousse à rechercher l'autonomie du pays. Mais il faut aussi empêcher la hausse des prix à la consommation, car assurer l'alimentation à un prix raisonnable permet de tranquilliser la population quand les libertés politiques limitées sont source de frustration. La protection de l'environnement est devenue un enjeu d'importance, en particulier la qualité de l'air dans les villes, mais aussi une vertu à afficher dans les relations internationales. Et brutalement, la crise de la PPA a conduit la Chine à s'ouvrir encore plus largement aux importations de viande et à " alléger " les obligations environnementales mises naguère à l'installation des élevages. Les politiques publiques sont en balancement, fréquent voire permanent, entre tenir le cap que l'on s'est fixé pour le long terme, en tout cas dans les intentions et les annonces, et s'adapter face aux évènements de diverses natures. S'adapter, ça peut être accepter de dévier provisoirement de son cap face à la survenue d'un accident majeur. Mais ça peut aussi signifier tergiverser en permanence et ne faire que des choix de court terme, en réaction à l'évolution du contexte. Ces variations sont incompatibles avec le respect d'une stratégie de long terme. La reconnaissance politique, donc les votes, est en partie déterminée par des résultats immédiats. Mais en matière d'alimentation, d'agriculture et d'élevage, le « juste " équilibre entre les multiples défis, le climat et l'environnement, la santé animale, l'alimentation de toute la population, le développement économique, l'efficacité et la compétitivité suppose les bons outils d'appréciation et de pilotage de la multiperformance (responsabilité de la recherche aussi) et une mise en œuvre déterminée sur le long terme qui est sous la responsabilité des décideurs publics.

\section{La recherche sur les systèmes d'élevage}

On est frappé par le gouffre vertigineux qui se creuse entre les images des projets chinois que Jean-Christophe Audonnet nous a montrées et les attentes souvent exprimées dans l'opinion française d'un élevage en plein air ou avec accès au plein air. Les motivations sont diverses : naturalité, respect du comportement spontané des animaux, effets supposés sur la qualité des produits... Sur quels systèmes d'élevage faut-il miser pour demain ? Les attentes et les critères d'appréciation évoluent rapidement alors que les éleveurs, pour investir, et ceux qui les accompagnent, pour les mises au point technologiques et les divers supports normatifs et financiers, ont besoin de se projeter sur au moins un cycle d'investissements. Et pour cela, même dix ans c'est court. L'Académie d'Agriculture entend bien se projeter dans ces visions d'avenir. La section "Productions animales " a un programme de travail en cours avec plusieurs auditions sur "l'Evolution des pratiques et des conditions d'élevage en bâtiment ". Cette section est aussi en train de reformuler ses orientations et son programme de travail à plus long terme autour de systèmes d'élevage d'avenir qui pourraient concilier l'offre suffisante de produits, la compétitivité, le respect de l'environnement, le respect des animaux... et bien sûr la santé animale. Pour cela, elle collabore avec toutes les autres sections de l'Académie, autant dans les disciplines techniques que socio-économiques, car le mot « système " est déterminant, non seulement à l'échelle de l'élevage, mais aussi de l'exploitation, des territoires. Et le système croise toutes les dimensions.

\section{CONCLUSION}

Sans faire d'amalgame, on voit que ces deux grands fléaux de santé publique dont nous parlons, la Covid19 en général et la Peste Porcine Africaine dans le monde de l'élevage, interrogent le « système monde " construit par les humains. Ils font partie du même système à tel point que leurs effets se croisent, s'amplifient mutuellement et parfois s'annihilent. A l'échelle du monde, santé humaine et santé animale, One Health, conditionneront l'avenir de nos activités. Cela appelle évidemment à de belles collaborations entre Académie Vétérinaire de France et Académie d'Agriculture de France.

\section{REMERCIEMENTS}

Les auteurs remercient Michel Thibier pour sa contribution à la préparation scientifique de la séance et Madame Ledoux (AAF) et Agreenium pour l'organisation de la visioconférence. 


\section{BIBLIOGRAPHIE}

- Bech-Nielsen S, Fernandez J, Martinez-Pereda F, Espinosa J, Perez Bonilla Q, Sanchez-Vizcaino JM. A case study of an outbreak of African swine fever in Spain. Br Vet J. 1995;151:203-214.

- Bernard J, Hutet E, Paboeuf F, Randriamparany T, Holzmuller P, Lancelot R, et al. Effect of O. porcinus Tick Salivary Gland Extract on the African Swine Fever Virus Infection in Domestic Pig. PLoS One. 2016;11:e0147869.

- Blueprint and Roadmap on the possible development of a vaccine for African Swine Fever prepared by the African Swine Fever EU reference laboratory1 on Commission request, Unit D4 Food safety programme, emergency funding. 2017. Disponible à : cff_animal_vet- progs_asf_blue-print-road-map.pdf (europa.eu) (consulté le 23.02.2021)

- Ebert A, Porkbarreling Pigs in Haiti. North American "Swine Aid" an Economic Disaster for Haitian Peasants, The Multinational Monitor. 1985. Disponible à : https://www.multinatio n a $1 \mathrm{monitor}$ org/hyper/ issues/1985/12/ebert-porkbarrel.html (consulté le 09.02.2021).

- IPBES, Workshop Report on Biodiversity and Pandemics of the Intergovernmental Platform on Biodiversity and Ecosystem Services, IPBES secretariat, Bonn, Germany, 2020. doi:10.5281/ zenodo.4147317

- Karger A, Pérez-Núñez D, Urquiza J, Hinojar P, Alonso C, Freitas FB, et al.
An Update on African Swine Fever Virology. Viruses. 2019;11:864.

- Lacasta A, Ballester M, Monteagudo PL, Rodríguez JM, Salas ML, Accensi F et al. Expression library immunization can confer protection against lethal challenge with African swine fever virus. J Virol. 2014;88:13322-13332.

- VACDIVA, A safe DIVA vaccine for African Swine Fever control and eradication A new vaccine for African swine fever, European Union, Funded under H2020-EU.3.2.1.1. 2019. Disponible à : A safe DIVA vaccine for African Swine Fever control and eradication | VACDIVA Project | H2020 | CORDIS | European Commission (europa.eu) (consulté le 23.02.2021). 